\title{
Uniform decrease of alpha-global field power induced by intermittent photic stimulation of healthy subjects
}

\author{
R. $\mathrm{Rau}^{1}$, \\ C. Raschka² and \\ H.J. Koch ${ }^{3}$
}

\author{
1Public Health Department, D istrict Wesel, M oers, Germany \\ ${ }^{2}$ Institute of Sports Sciences, J.-W. Goethe University, Frankfurt, \\ Germany \\ ${ }^{3}$ Department of Gerontopsychiatry, University H ospital of Psychiatry, \\ Regensburg, Germany
}

\section{Correspondence \\ H.J. Koch \\ Department of Gerontopsychiatry \\ University Hospital of Psychiatry \\ Universitätsstrasse 84 \\ D-93053 Regensburg \\ Germany \\ Fax: + 49-941-941-1205 \\ E-mail: horst.koch@ bkr-regensburg.de

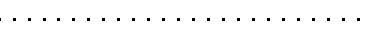

Received March 29, 2001

Accepted February 25, 2002

\section{Abstract}

Nineteen-channel EEGs were recorded from the scalp surface of 30 healthy subjects (16 males and 14 females, mean age: 34 years, SD: 11.7 years) at rest and under trains of intermittent photic stimulation (IPS) at rates of 5, 10 and $20 \mathrm{~Hz}$. Digitalized data were submitted to spectral analysis with fast fourier transformation providing the basis for the computation of global field power (GFP). For quantification, GFP values in the frequency ranges of 5,10 and $20 \mathrm{~Hz}$ at rest were divided by the corresponding data obtained under IPS. All subjects showed a photic driving effect at each rate of stimulation. GFP data were normally distributed, whereas ratios from photic driving effect data showed no uniform behavior due to high interindividual variability. Suppression of alpha-power after IPS with $10 \mathrm{~Hz}$ was observed in about $70 \%$ of the volunteers. In contrast, ratios of alpha-power were unequivocal in all subjects: IPS at $20 \mathrm{~Hz}$ always led to a suppression of alpha-power. Dividing alpha-GFP with $20-\mathrm{Hz}$ IPS by alpha-GFP at rest $\left(\mathrm{R}=\alpha-\mathrm{GFP}_{\mathrm{IPS}} / \alpha-\mathrm{GFP}_{\text {rest }}\right)$ thus resulted in ratios lower than 1 . We conclude that ratios from GFP data with $20-\mathrm{Hz}$ IPS may provide a suitable paradigm for further investigations.

\section{Introduction}

The first studies on alterations of electric cerebral activity induced by intermittent photic stimulation (IPS) were done by Adrian and Matthews in 1934 (see 1). It was observed that brain waves can be synchronized by external stimulation with rhythmic flashlights, a phenomenon which is pronounced above occipital brain regions. This phenomenon was called photic driving effect (PDE). In this physiological reaction interindividual variability is high. In some cases EEG activ- ity coupling to stimulation rates does only or most visibly occur at frequencies corresponding to the spontaneous rhythm, the so-called "frequency selectivity" (1,2). EEG changes evoked by photic stimulation at low frequencies correspond to single visually evoked potentials, while series of brain waves at higher stimulation rates are related to steadystate visually evoked potentials.

Global field power (GFP) is currently a widely used technique in clinical neurophysiology and pathophysiology such as visual discrimination performance during saccades, 
epilepsy research or information processing studies $(3,4)$. Brain mapping results are influenced by drugs and medications (5). Michel et al. (6) reported that GFP is influenced by diazepam and that the extent of influence depends on the functional state of the central nervous system. GFP analysis can serve to monitor treatment of Alzheimer's disease with tacrine or related drugs (7). The ratio of alpha- versus delta-GFP shows a positive correlation with tau protein levels in patients with cognitive decline due to supposed Alzheimer's disease (8). In cerebrovascular diseases an increase of PDE was observed and vertebrobasilar perfusion insufficiency led to a reinforced PDE in the $\beta$ frequency range (1). Alterations of $\mathrm{PDE}$ were described in patients with migraine and other forms of headache (9). Apart from PDE, the phenomenon of alpha-suppression has been explored by several investigators, who reported differences between headache and migraine patients: IPS at rates of $20 \mathrm{~Hz}$ in patients suffering from migraine did not lead to the alpha-suppression which occurs in healthy volunteers. In some patients IPS elicited even an activation of brain waves similar to PDE (10-15).

The objective of the present study was to assess whether $20-\mathrm{Hz}$ IPS elicits a stable decrease of GFP at different frequency bands in healthy volunteers. If such a systematic alteration could be ascertained, this finding could serve as a useful experimental paradigm in future studies.

\section{Material and Methods}

EEGs of 30 healthy subjects (16 males and 14 females; mean age $=34$ years, $\mathrm{SD}=$ 11.7 years, range: $21-51$, median $=31$ ), from whom informed consent was obtained beforehand, were recorded at rest for $40 \mathrm{~s}$ and under IPS series of $30 \mathrm{~s}$ each at 5, 10 and 20 $\mathrm{Hz}$. White flashlight stimulation (single flashes, $0.5 \mathrm{~J} /$ flash) was applied with a stroboscope placed at a distance of $20 \mathrm{~cm}$ in front of the subjects who kept their eyes closed. Each train of flashlight stimulation was followed by an interval of $20 \mathrm{~s}$. The recording was performed in a darkened, sound-proof and climatized room between 11 and $12 \mathrm{am}$. The subjects lay in a comfortable recumbent position.

EEGs were recorded with 19 surface electrodes arranged according to the international ten-twenty-system, with a time constant of $0.3 \mathrm{~s}$ and a low-pass filter of $70 \mathrm{~Hz}$. Frequency of digitalization was $512 \mathrm{~Hz}$. Data were digitalized and computed by fast fourier transformation (FFT) for spectral analysis and brain mapping representation. This method, also known as EEG or brain mapping, is a computer-assisted analysis of the conventional EEG. Brain waves recorded from the scalp with surface electrodes are amplified in a first step. At the same time, analogous signals are transformed into digital data, i.e., voltage curves from each EEG electrode are recorded with a programmed frequency and are saved for subsequent offline analysis by means of FFT, and spectra of frequency or amplitude can be calculated (16). These spectra show the distribution of amplitudes and frequencies of brain waves during a certain time interval for a single electrode. Data from single electrodes can be integrated by way of interpolation of $\mathrm{C}$ electrodes of an array $\mathrm{A}$ as described by Brandeis et al. (17):

$$
\operatorname{GFP}(A)=\left[1 / \mathrm{C} * \sum_{\mathrm{C}=1}^{\mathrm{C}} \mathrm{A}_{\mathrm{c}}^{2}\right]^{1 / 2}
$$

The GFP can be considered as a reference-free characteristic measure of the hilliness (i.e., standard deviation of potential at electrodes with regard to an average reference) of a potential landscape calculated from EEG data. A low GFP reflects a flat potential distribution, i.e., desynchronization of neural activity, with few peaks and troughs, whereas a high GFP stands for a potential distribution with many peaks and 
troughs. GFP values for separate frequency bands can be calculated. The topographic distribution of activity (power spectrum, $\mu \mathrm{V}^{2}$ ) above the scalp surface can be displayed in various forms $(1,18)$. Data from power analysis can be visualized in the form of twodimensional color-coded brain maps (19). In addition, GFP dissimilarity (range 0 to 1 ) which compares two successive maps and is inversely related to the stability of potentials was calculated (17).

From each phase 20-s EEG recordings (i.e., rest versus trains of IPS) were obtained for spectral analysis. Artifacts due to muscle contraction, twinkling, inspiration, etc., were excluded in the off-line analysis. Data analysis focused on four frequency bands: firstly ranges were defined according to the stimulation frequencies $\pm 10 \%$, i.e., the 5 -Hz frequency ranged from 4.5 to $5.5 \mathrm{~Hz}$; the same procedure was applied to the $10-\mathrm{Hz}$ and $20-$ $\mathrm{Hz}$ range. Secondly, the alpha range was confined to the area of 8 to $12 \mathrm{~Hz}$.

The GFP data for 30 healthy volunteers at rest were compared to those obtained during IPS at 5, 10 and $20 \mathrm{~Hz}$. In order to quantify $\mathrm{PDE}, \mathrm{R}$ ratios were computed from GFP data at rest in comparison with those obtained from EEGs under IPS, i.e., $\mathrm{R}=$ $\alpha-G_{\text {GFP }} / \alpha-G_{\text {IPP }}$ rest All data were analyzed qualitatively and are presented as box plots and histograms. T-value maps were computed according to the method of Duffy (20). Moreover, nonparametric Wilcoxon tests for related samples were applied for comparison of alpha-GFP values at rest and under stimulation (21).

The protocol was approved by the Hospital Ethics Committee.

\section{Results}

All subjects showed normal EEG results, which could be allocated to classical EEG categories and PDE was observed in all cases. Figure 1 shows original color-coded power maps of different frequency bands of a single volunteer for different frequency bands at rest and for different photic stimulation conditions. Corresponding GFP values and dissimilarities were calculated. GFP at rest and under IPS displayed a high interindividual variability within defined frequency ranges but the data showed normal distribution (Figure 2A-C). Generally, the highest GFP values were found for the $10-\mathrm{Hz}$ range, independently of the IPS conditions. Due to this marked variability of trends in flashlightinduced changes no stable ratio of GFP values could be calculated in this context. In contrast, alpha-GFP ratios yielded more uniform results. Whereas activity under $10-\mathrm{Hz}$ IPS produced inconsistent changes with reduction of alpha-power in approximately $70 \%$ of the subjects, intermitted photic stimulation with 20 flashes per second led in all cases to a homogenous desynchronization of waves which was expressed as a reduction of alpha-GFP.

The $\mathrm{R}$ ratio of alpha-GFP under $20-\mathrm{Hz}$ IPS and alpha-GFP at rest resulted in values lower than 1 (Figure 3). Eighteen of 30 volunteers showed ratios within the 0.8 and 1.0 range. The corresponding box plots are given in Figure 4 and show a difference of median GFP values of the order of 2. GFP differences between values at rest and under IPS were highly significant $(\mathrm{P}<0.001)$ as proven by a Wilcoxon test for matched pairs. $T$-test analysis of spectral data above single electrodes (so-called $t$-value mapping) revealed that power reduction was most pronounced in the parieto-occipital region.

\section{Discussion}

In contrast to the results of previous studies $(1,14)$, all 30 subjects in the present investigation showed PDE with a high interindividual variability. Even in EEG samples with only mild photic driving, spectral analysis rendered visible peaks of electric field power in the frequency range that corresponded to the stimulation rate and, in addi- 

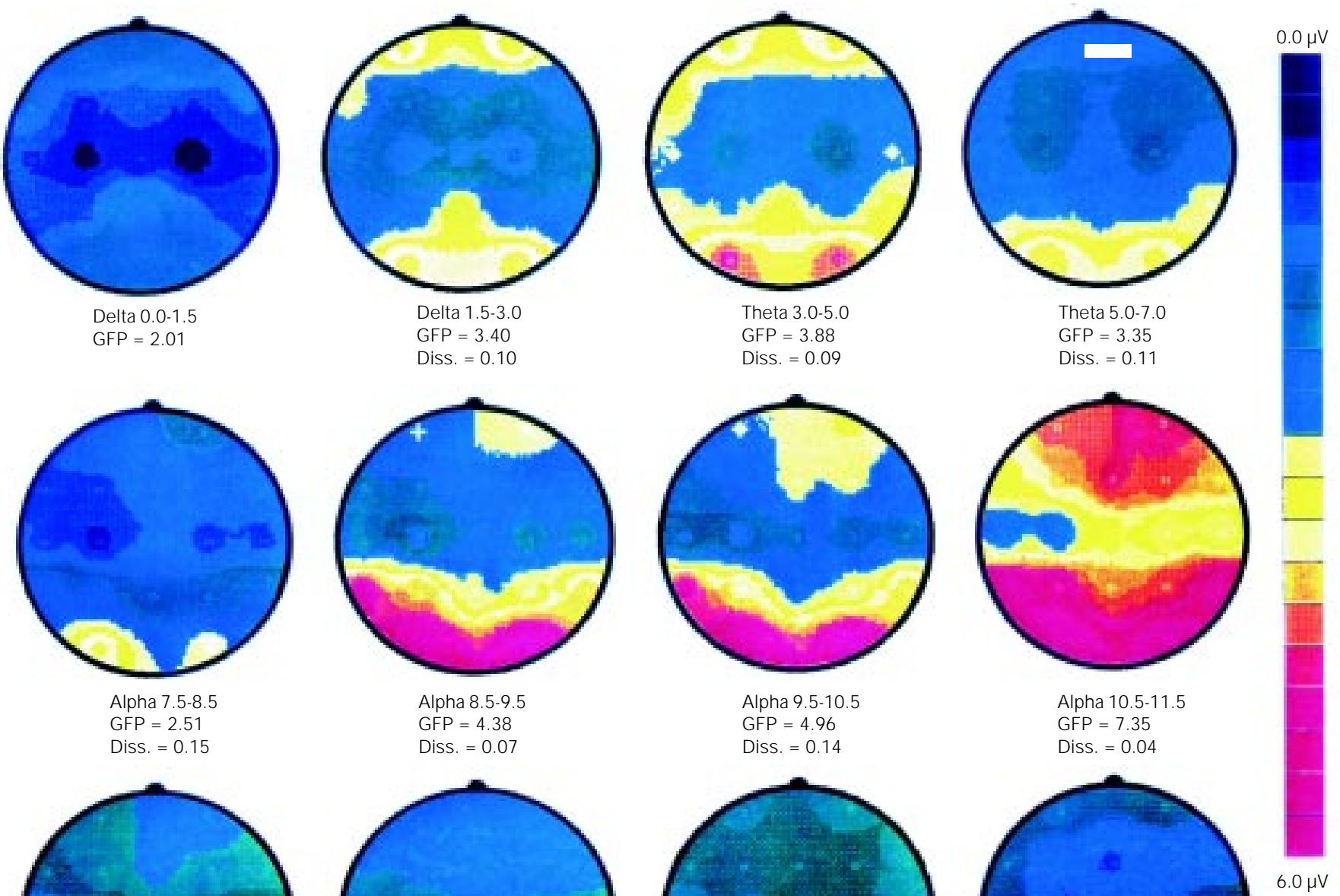

Alpha 7.5-8.5

Alpha 8.5-9.5

GFP $=2.51$

GFP $=4.38$

Diss. $=0.07$
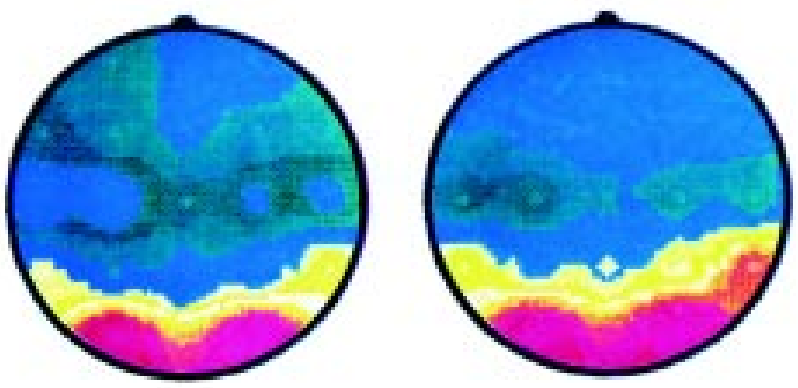

Alpha 9.5-10.5

GFP $=4.96$

Diss. $=0.14$

Alpha 10.5-11.5

GFP $=7.35$

Diss. $=0.04$
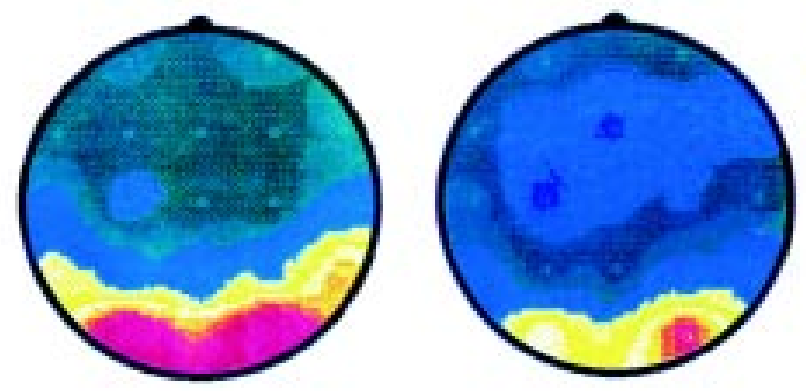

Alpha 12.5-13.5

GFP $=4.91$

GFP $=4.23$

Diss. $=0.07$

Diss. $=0.02$

Beta 13.0-18.0

GFP $=3.66$

Diss. $=0.16$
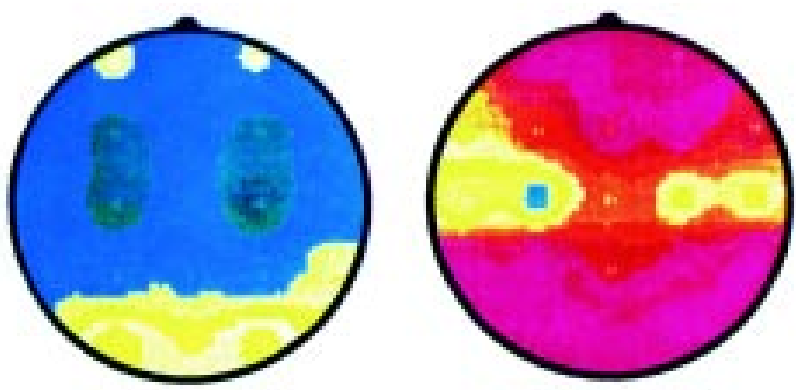

fs5

GFP $=3.50$

fs 10

GFP $=8.19$

Diss. $=0.16$

Diss. $=0.34$

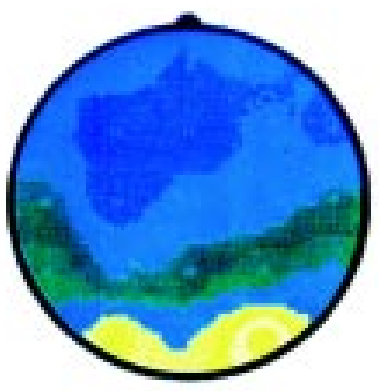

fS20

$\mathrm{GFP}=2.47$

Diss. $=0.22$
Beta 18.0-30.0

GFP $=2.81$

Diss. $=0.11$

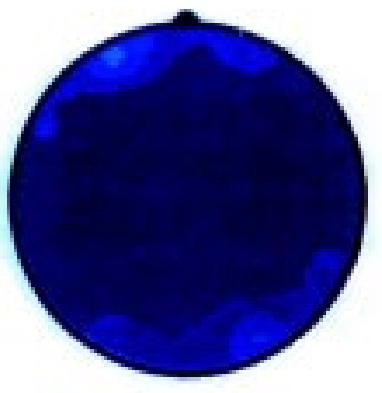

fs30

GFP $=0.92$

Diss. $=0.31$

Figure 1. Power maps showing activity distribution for delta-, theta-, alpha- and beta-ranges (row 1 to row 3) of a single individual (female, 29 years). The brain maps in the fourth row represent the analyzed frequency ranges corresponding to stimulation rates (fs) of 5, 10, 20 and $30 \mathrm{~Hz}$. The corresponding global field power (GFP) and dissimilarity (Diss.) values are given below the respective maps. 


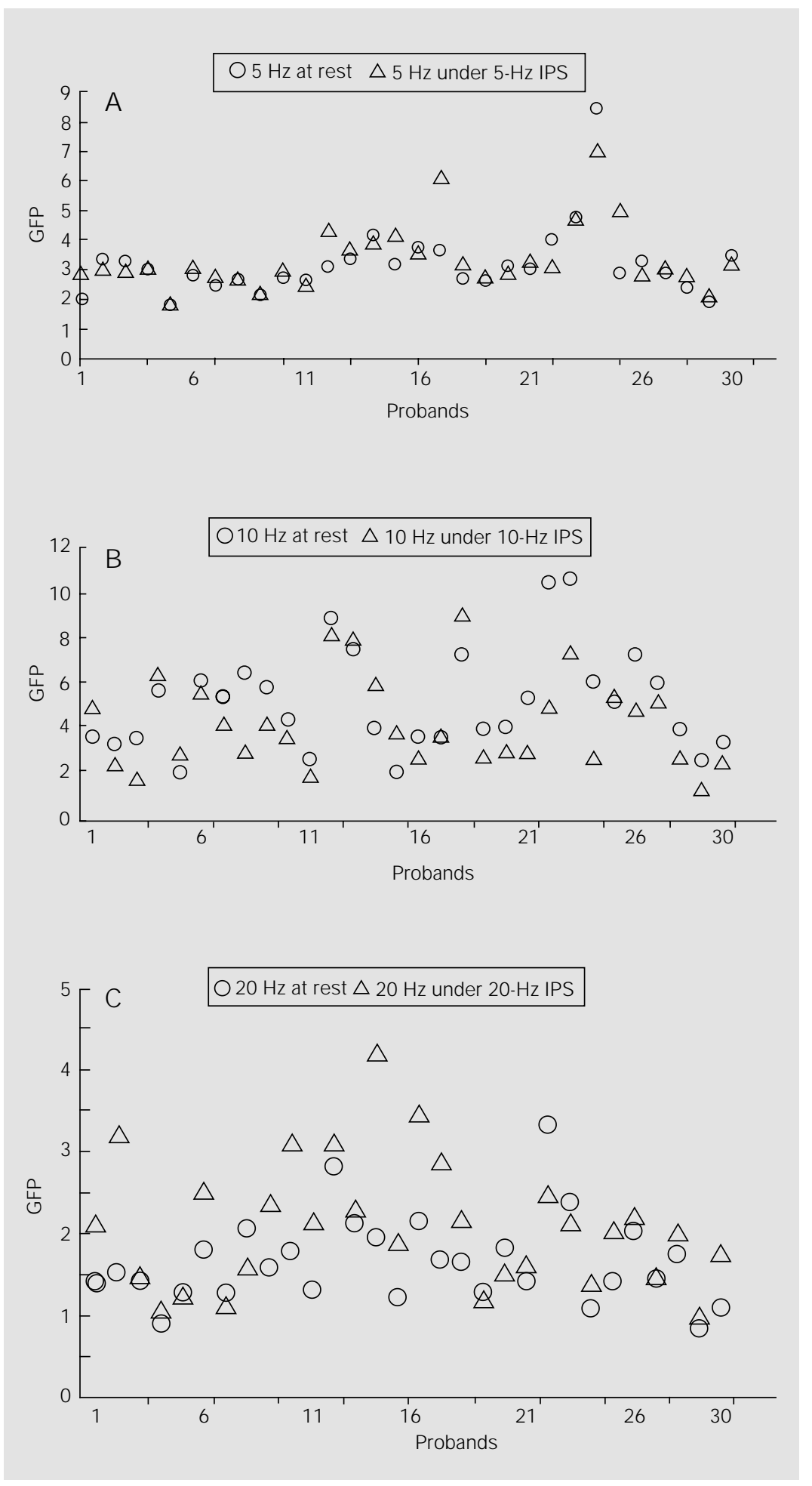

Figure 2. A, Comparison of global field power (GFP) values in the 5-Hz range at rest and during intermittent photic stimulation (IPS) with $5 \mathrm{~Hz}$ of 30 subjects. B, Comparison of GFP values in the $10-\mathrm{Hz}$ range at rest and during IPS with $10 \mathrm{~Hz}$ of 30 subjects. C, Comparison of GFP values in the 20-Hz range at rest and during IPS with $20 \mathrm{~Hz}$ of 30 subjects. 
Figure 3. Distribution of ratios of alpha-global field power following intermittent photic stimulation with $20 \mathrm{~Hz}$ and at rest in 30 healthy volunteers $(P<0.001$, Wilcoxon test).

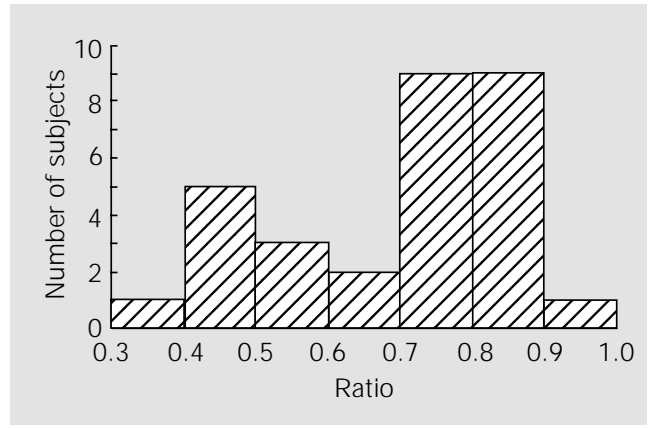

Figure 4. Box plots including median and 25 or $75 \%$ percentiles or non-outlier extreme values of alpha-global field power (GFP) results at rest and under intermittent photic stimulation (IPS) with $20 \mathrm{~Hz}$ in 30 healthy subjects.

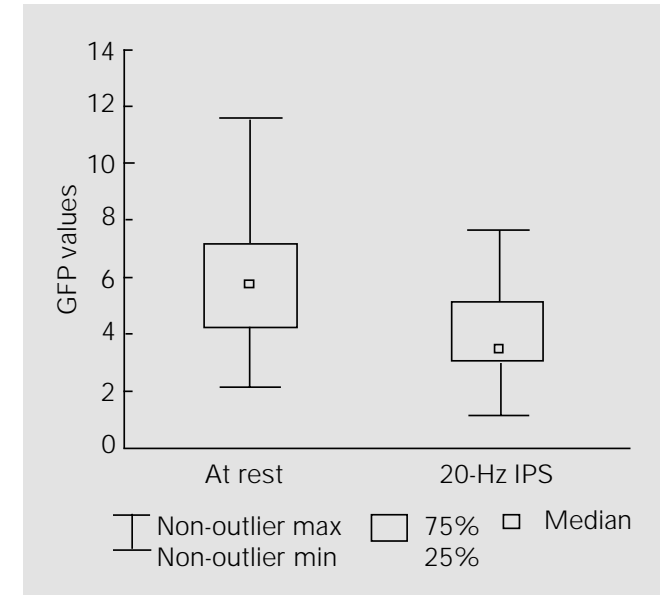

tion, partially revealed harmonics. In accordance with earlier studies (11) which found suppression by photic and acoustic stimulation, we observed a reduction of GFP in the $10-\mathrm{Hz}$ domain under IPS in most cases (70\%).

As the essential result of our study, we found that GFP consistently decreased under the influence of $20-\mathrm{Hz}$ IPS, i.e, GFP decreased below the initial level (GFP at rest) in all cases. The calculated GFP ratio proved to be a stable parameter in EEG diagnosis, with this ratio being smaller than 1 in all subjects. Previous investigations on healthy persons and headache or migraine patients compared local EEG changes above single electrodes $(10,12,15)$ and revealed a decrease of power at single occipitoparietal electrodes induced by $20-\mathrm{Hz}$ IPS in normal healthy volunteers. In the present study, GFP was employed as a criterion which integrates data gained from all surface electrodes. Nonetheless, attenuation of alpha-power led to a uniform decrease of GFP in the alpha-range compared to the initial data, indicating general desynchronization of brain activity. We conclude that, with regard to physiological occipital alpha-dominance, GFP as a global and general parameter reliably reflects local changes induced by IPS in the alpha-range and may thus be a practical and suitable instrument in daily clinical practice.

Further investigation will be needed to obtain comparable ratios from patient EEG data. Within this context, migraine and headache patients will be of special interest, since previous studies along with preliminary findings in our clinical experience suggest that migraine patients show significantly different electrophysiologic patterns of reaction to IPS with a flashlight in comparison to healthy controls $(10,12,15)$. Within this context, missing suppression or even an activation of brain waves during $20-\mathrm{Hz}$ IPS is to be expected in migraine patients.

A ratio lower than 1 may be an indicator of the disturbance of neuronal excitability of the visual cortex common in migraine $(1,10,12,13,15)$. We conclude that the ratio obtained from GFP data may provide stable parameters and reproducible results. Further studies are needed to assess the clinical relevance of ratios and possible correlations between ratios and clinical features.

\section{References}

1. Zschocke S (1995). Klinische Elektroenzephalographie. Springer, Heidelberg, Germany, 211-221.

2. Mast J \& Victor J D (1991). Fluctuations of steady-state VEPs: interaction of driven evoked potentials and the EEG. Electro- encephalography and Clinical Neurophysiology, 78: 389-401.

3. Anagnostou E, Kleiser R \& Skrandies W (2000). Electrophysiological correlates of human intrasaccadic processing. Experimental Brain Research, 130: 177-187.
4. Skrandies W, Reik P \& Kunze C (1999). Topography of evoked brain activity during mental arithmetic and language tasks: sex differences. Neuropsychologia, 37: 421-430.

5. Crocq MA, Beaubernard C, Minot R, 
Deliry J -L \& Macher J -P (1989). Application de l'EEG quantitative et de la cartographie EEG à l'étude des psychotropes. In: Rondot P, Gaches J \& Sebban C (Editors), Cartographie EEG: Méthodologie et Applications Cliniques. Editions Médicales. Frison-Roche, Paris, France, 207216.

6. Michel CM, Pascual-Marqui RD, Strik WK, Koenig T \& Lehmann D (1995). Frequency domain source localization shows statedependent diazepam effects in 47-channel EEG. J ournal of Neural Transmission (Genetic Section), 99: 157-171.

7. J elic $V$, Dierks $T$, Amberla $K$, Almkvist $O$, Winblad B \& Nordberg A (1998). Longitudinal changes in quantitative EEG during long-term tacrine treatment of patients with Alzheimer's disease. Neuroscience Letters, 254: 85-88.

8. J elic V, Blomberg M, Dierks $T$, Basun $H$, Shigeta $M$, J ulin $P$, J ensen $M$, Lannfelt $L$, Winblad B \& Wahlund LO (1998). EEG slowing and cerebrospinal fluid tau levels in patients with cognitive decline. NeuroReport, 9: 157-160.

9. Klotz J M, Heils A \& Langohr HD (1992). Quantitative und topographische Analyse des Photic-Driving-Effektes bei Patienten mit Migraine. Nervenheilkunde, 11: 316322.

10. Genco S, de Tommaso M, Prudenzano AM, Savarese M \& Puca FM (1994). EEG features in juvenile migraine: topographic analysis of spontaneous and visual evoked brain electrical activity: a comparison with adult migraine. Cephalalgia, 14: 41-46.

11. Grillon C \& Buchsbaum MS (1986). Computed EEG-topography of response to visual and auditory stimuli. Electroencephalography and Clinical Neurophysiology, 63: 42-53.

12. Puca FM, de Tommaso $M$, Tota $P$ \& Sciruicchio V (1996). Photic driving in migraine: correlations with clinical features. Cephalalgia, 16: 246-250.

13. Scheuler W (1983). Zur klinischen Bedeutung der gesteigerten Photostimulationsreaktion im Alpha-Frequenzbereich. Zeitschrift für Elektroenzephalographie, Elektromyographie und Verwandte Gebiete, 14: 143-153.

14. Takahashi T (1993). Activation methods. In: Niedermeyer E \& Lopes da Silva F (Editors), Electroencephalography. 3rd edn. Williams \& Wilkins, Baltimore, MD, USA, 241-262.

15. Tsounis $S \&$ Varfis G (1992). Alpha rhythm power and the effect of photic stimulation in migraine with brain mapping. Clinical Electroencephalography, 23: 1-6.

16. Brandl U \& Wenzel D (1989). Advanced programming techniques for activity mapping on personal computers. In: Maurer K (Editor), Topographic Brain Mapping of EEG and Evoked Potentials. Springer, Heidelberg, Germany, 129-135.

17. Brandeis $D$, Lehmann D, Michel CM \& Mingrone D (1995). Mapping event-related brain potential microstates to sentence endings. Brain Topography, 8: 1-15.

18. Lehmann D \& Skrandies W (1980). Reference-free identification of components of checkerboard-evoked multichannel potential field. Electroencephalography and Clinical Neurophysiology, 48: 609-621.

19. Klotz J M (1993). Topographic EEG mapping methods. Cephalalgia, 13: 45-52.

20. Duffy FH (1989). Topographic mapping of brain electrical activity: Clinical applications and issues. In: Maurer K (Editor), Topographic Brain Mapping of EEG and Evoked Potentials. Springer, Heidelberg, Germany, 19-52.

21. Zar JH (1984). Biostatistical Analysis. Prentice Hall International Edition, Englewood Cliffs, NJ , USA. 